\title{
An Update of Long-Noncoding RNAs in Acute Kidney Injury
}

\author{
Lina Yang ${ }^{\dagger}$, Bo Wang ${ }^{\dagger}$, Liang Ma* and Ping Fu* \\ Kidney Research Institute, Division of Nephrology, West China Hospital of Sichuan University, Chengdu, China
}

Acute kidney injury (AKI) is a global public health concern with high morbidity, mortality, and medical costs. Despite advances in medicine, effective therapeutic regimens for AKI remain limited. Long non-coding RNAs (IncRNAs) are a subtype of non-coding RNAs, which longer than 200 nucleotides and perform extremely diverse functions in biological processes. Recently, IncRNAs have emerged as promising biomarkers and key mediators to AKI. Meanwhile, existing research reveals that the aberrant expression of IncRNAs has been linked to major pathological processes in AKI, including the inflammatory response,

OPEN ACCESS

Edited by:

Carolyn Mary Ecelbarger,

Georgetown University, United States

Reviewed by:

Xiao-ming Meng,

Anhui Medical University, China

Lin Sun,

Central South University, China

*Correspondence:

Liang Ma

liang_m@scu.edu.cn

Ping Fu

fupinghx@scu.edu.cn

${ }^{\dagger}$ These authors have contributed equally to this work and share first

authorship

Specialty section:

This article was submitted to Renal and Epithelial Physiology, a section of the journal

Frontiers in Physiology

Received: 06 January 2022

Accepted: 15 February 2022

Published: 08 March 2022

Citation:

Yang $L$, Wang $B, M a L$ and Fu P (2022) An Update of LongNoncoding RNAs in Acute Kidney

Injury.

Front. Physiol. 13:849403.

doi: 10.3389/fphys.2022.849403 cell proliferation, and apoptosis, via forming the IncRNA/microRNA/target gene regulatory axis. Following a comprehensive and systematic search of the available literature, 87 relevant papers spanning the years 2005 to 2021 were identified. This review aims to provide and update an overview of IncRNAs in AKI, and further shed light on their potential utility as AKI biomarkers and therapeutic targets.

Keywords: acute kidney injury, long non-coding RNA, sepsis, ischemia-reperfusion, post-contrast AKI, kidney transplant, drug induced AKI

\section{INTRODUCTION}

Acute kidney injury (AKI) is a severe clinical syndrome characterized by an abrupt decrease in kidney function, as measured by high blood creatinine and decreased urine volume within 7 days (Kellum et al., 2012). Epidemiologic studies have found that about 13.3 million people worldwide suffer from AKI each year (Mehta et al., 2015). Furthermore, the global health burden of AKI-related mortality is far beyond that of breast cancer, heart failure, or diabetes (Lewington et al., 2013). Therefore, finding effective prevention and treatment approaches are indispensable. Clinically, the cause of AKI can be divided into three categories: pre-renal (impaired renal perfusion), intra-renal, and post-renal (urinary tract obstruct). Among these, sepsis, and ischemia/reperfusion (I/R) are the common leading causes of AKI. Besides, postcontrast AKI (CI-AKI), transplant-related AKI, and drug or toxin induced AKI have become prevalent and important as medical care evolves as well. The pathogenesis of AKI is multifactorial and has been overwhelmingly implicated in the etiology of AKI (Kinsey and Okusa, 2011).

Even though our understanding of the pathophysiology of AKI is continually expanding, the precise processes remain unknown. Following the improvements and cost reductions of high throughput RNA-sequencing, considerable research has reported that the non-coding RNAs (ncRNAs), especially long non-coding RNAs (lncRNAs), are strongly associated with the pathophysiological process underlying AKI. As the quantity of research on lncRNAs has grown fast, in order to offer a thorough and up-to-date overview of the function of lncRNAs in AKI, we briefly introduce the lncRNA and systematically review the mechanism of functional 
lncRNAs in AKI due to various etiologies. Finally, we highlight certain aspects of the lncRNA mechanism in AKI, as well as future research directions.

\section{OVERVIEW OF LNCRNAS}

LncRNAs are a subtype of ncRNA that longer than $200 \mathrm{nt}$ with no protein-coding potential and make up the most considerable portion of the mammalian non-coding transcriptome (Mercer et al., 2009; Derrien et al., 2012). Sequence analysis of $\operatorname{lncRNAs}$ between different species revealed that lncRNAs presented the characteristics of low DNA sequence conservation and low expression. Commonly, according to lncRNA location concerning protein-coding genes, lncRNAs could be classified as either intergenic or intragenic. Intergenic lncRNAs do not intersect with any protein-coding genes. In contrast, intragenic lncRNAs overlap with protein-coding genes and could be further sorted into antisense, bidirectional, intronic, and overlapping sense lncRNAs. In parallel, IncRNAs could also be categorized based on their length, location, transcript properties, regulatory mechanisms, function, and so on (Statello et al., 2021).

Most lncRNA species are transcribed by Pol II. It also could be capped, spliced, and polyadenylated, exported like mRNA. However, after being transcribed by Pol II, lncRNAs will further fold into the complex secondary or tertiary structures. Those high-ordered structures showed higher conserved than their primary sequence. Diversified structure enables lncRNAs to participate in life activities and disease progress by interacting with DNA, RNA, or protein to regulate gene expression at multiple levels (epigenetic regulation, transcription regulation, and post-transcriptional regulation). Among these, research on the roles of lncRNAs in post-transcriptional regulation has been investigated the most. Three major mechanisms of lncRNA regulate the gene expression in post-transcriptional: a. binding to RNA or structures to form a lncRNA-protein complexes directly; b. pairing with the other RNAs to recruit proteins; c. competing endogenous RNAs or 'sponges' of miRNAs to restrict miRNA availability to target mRNAs (Marchese et al., 2017; Salviano-Silva et al., 2018). Aside from the structure, the location of lncRNAs in cells also exerts an intensive effect on their functions (Chen, 2016). In addition to being located in the nucleus, IncRNAs have been detected in additional subcellular sites such as mitochondria and exosomes (Bridges et al., 2021). Moreover, these organelle-specific lncRNAs are intensively implicated in organelle homeostasis, which is always plays a role in the occurrence and development of the disease (Li et al., 2021b).

Currently, research methodologies for investigating the functions of lncRNAs in disease include lncRNA identification, lncRNA characteristic analysis, functional investigations, and molecular processes (Gao et al., 2020a). Similarly, advances in experimental equipment and computational methodologies in lncRNAs study have occurred in recent years. Among these advancements, fully functional sequencing databases stands out. First, with the introduction of third generation sequencing technology and lncRNAs databases, lncRNA screening has grown completer and more reliable. Second, emerging bioinformatics algorithm, database RACE, and fluorescence in situ hybridization (FISH) offer great assistance in annotating the characteristic of lncRNAs, such as coding potential, location information on the genome, secondary structure, correlation with the diseases, full-length analysis, and cell localization (Liu et al., 2018). Then, the roles of lncRNAs are generally validated by overexpression or Knock down of specific lncRNAs, and their role in diseases development is studied by assessing changes in biological behaviors in vitro and in vivo tests. Finally, by combining the prediction work of databases and experiment technology such as DNA-FISH (Gelali et al., 2019), chromatin immunoprecipitation (Nakato and Sakata, 2021), RNA-pull down, luciferase reporters (Cao et al., 2019), and methylationspecific PCR (Hernández et al., 2013), the specific molecular regulation network between the protein, RNA, or DNA, and the lncRNAs could be illustrated.

Based on the strategies and technologies mentioned above, studies have identified that lncRNAs serve a crucial role in various biological processes, ranging from the cell cycle, growth, apoptosis, and immune responses to some tissue-specific physiology. Many lncRNAs have been verified to have links with prevalent diseases, such as cancer, neurological and cardiovascular diseases (Fenoglio et al., 2013; Peng et al., 2017; Fernandes et al., 2019). Simultaneously, increased emphasis has been placed on the significance of lncRNAs in renal disorders.

\section{LNCRNAS IN SEPTIC AKI}

\section{LncRNAs Globally Changed in Septic AKI}

Patients who suffer from sepsis are associated with a higher risk of incidence and mortality of AKI. The pathogenesis of sepsis-induced AKI is very complex and remains unclear. Mounting evidence suggests that lncRNAs could exhibit diverse functions in physiological and pathological processes of septic AKI (Figure 1, Table 1). The large-scale analyses showed that a significant expression difference of lncRNAs between AKI and normal control does exist, both in mice and humans. Chun-Mei and collaborators identified 5,361 up-regulated lncRNAs and 5,928 down-regulated lncRNAs differentially expressed in septic AKI humans compared with a control group (Chun-Mei et al., 2016). Another meta-analysis summarized the 38 independent studies and recognized 31 remarkably dysregulated lncRNAs in total, some of which have been considered the potential predictive biomarkers and therapeutic targets of AKI and further verified in the research as follows already (Ma et al., 2021).

\section{LncRNAs Promoting Septic AKI}

LncRNAs that are up-regulated in septic AKI are always a focus for investigation. As mentioned before, lncRNAs can act as competing endogenous RNAs to compete with mRNAs for binding to the same miRNAs and influence the expression of miRNA-targeted transcripts. The interplay between the lncRNAs, miRNAs, and mRNAs is called a ceRNA crosstalk. Constructing 


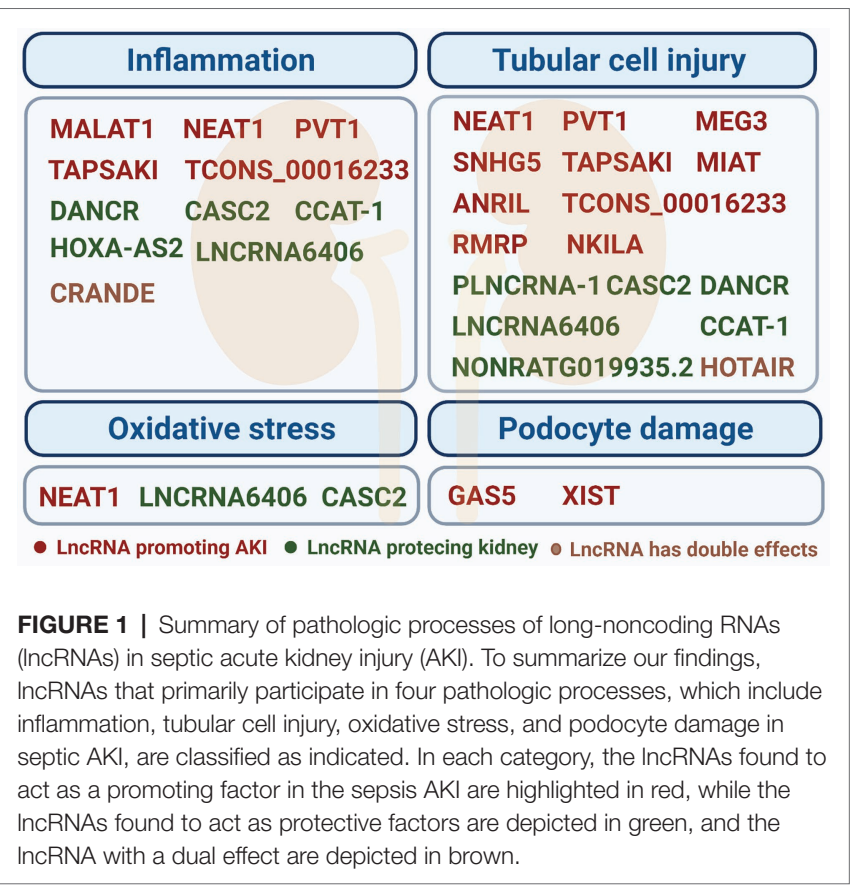

ceRNA regulatory networks is the most common method for studying the biological functions of lncRNAs (Lan et al., 2019). LncRNA metastasis-associated lung adenocarcinoma transcript 1 (MALAT1) is one of the well-studied lncRNA involved in septic AKI. The expression of MALAT1 is increased in sepsis patients' serum, experimental animal kidney tissue, and cell lines. MALAT1 could promote kidney injury via downregulating miR-146a and activating the nuclear factor- $\mathrm{\kappa B}(\mathrm{NF}-\kappa \mathrm{B})$ in LPS induced AKI. Correspondingly, silencing MALAT1 displayed a significant renal protective effect (Ding et al., 2018). Some medications, such as paclitaxel and dexmedetomidine, could also exert their therapeutic effect by reducing MALAT1 expression in tubule cells (Xu et al., 2020; Zhu and Lu, 2020a).

Numerous scholars have also intensively studied the function of long noncoding RNA nuclear-enriched abundant transcript 1 (NEAT1) in sepsis-induced AKI. Depletion of NEAT1 could attenuate sepsis-induced AKI via regulating the miR-22-3p/ NF- $\kappa B$ pathway (Feng et al., 2020). Furthermore, lower expression of NEAT1 may obstruct the development of LPS-induced injury and inflammation in HK-2 cells by targeting the let-7b-5p/ TRAF6 axis or miR-93-5p/TXNIP axis (Gao et al., 2020b; Yang et al., 2021b). siNEAT1 could alleviate the cecal ligation punctures (CLP)-induced AKI in rats via miR-27a-3p/TAB3 axis, presenting in reducing kidney injury, ameliorated renal function, inflammation, and cell death (Wang et al., 2020a). Moreover, downregulation of NEAT1 ameliorated LPS-induced inflammatory responses by promoting macrophage M2 polarization via the miR-125a-5p/TRAF6/TAK1 axis (Wang and Guo, 2020).

Another lncRNA plasmacytoma variant translocation 1 (PVT1) has also gained much attention. Suppression of PVT1 in LPS-treated HK-2 cells could inactivate the NF-KB pathway via miR-17-5p, accompanied by improved cell viability and reduced inflammatory response (Yuan et al., 2021b). Besides, the reduction of PVT1 is also the mechanism of how curcumin protects against septic kidney injury (Huang et al., 2020). Additionally, PVT1 knockdown inhibited LPS-induced cell pyroptosis targeting the miR-20a-5p/NLRP3 signaling pathway (Deng et al., 2021b). The IncRNA DLX6 antisense RNA1 (DLX6-AS1) and the function of lncRNA maternally expressed gene 3 (MEG3) could function as the enablers of cell pyroptosis in LPS-induced AKI by decreasing miR-223-3p and targeting MEG3/miR-21/PDCD4 axis and miR-18a-3p/GSDMD, respectively (Yang et al., 2018; Tan et al., 2020; Deng et al., 2021a).

Similar to the regulatory network between lncRNAs and miRNAs mentioned above, the connecting as follows could also play a role in promoting cell apoptosis or inflammation response in septic AKI by sponging the related miRNA, TapSAKI/ miR-22/PTEN/TLR4/NF- $\mathrm{kB}$ (Shen et al., 2019), TapSAKI/ miR-205/IRF3axis (Han et al., 2021b), SNHG14/miR-93 (Shi et al., 2021), SNHG14/miR-4953p/HIPK1 (Yang et al., 2021a), SNHG5/miR-374a-3p/TLR4/NF-kB (Wang et al., 2021), MIAT/ miR-29a (Zhang et al., 2019), ANRIL/miR-199a (Zhu et al., 2020b), TCONS_00016233/miR-22-3p (Zhang et al., 2020b), SIKIAT1/miR-96-3p/FOXA (Lu et al., 2020b), RMRP/miR-206 (Zhang et al., 2021), NKILA/miR-140-5p/CLDN2 (Han et al., 2021a), and XIST/miR-15a-5p/CUL3 (Xu et al., 2019).

Genes that expression elevated in AKI always play a role in promoting disease progression. Correspondingly, genes down-regulated exhibited the potential to suppress disease progression. There are, of course, exceptions. In the studies of podocyte injury of AKI, IncRNA growth arrest-specific transcript 5 (GAS5) expression decreased in a time-dependent manner, and GAS5 inhibition promoted podocyte injury by inhibiting the expression of PTEN via mediating the PI3K/ AKT pathway (Fang et al., 2018).

\section{LncRNAs Exerted Nephroprotective Effects on Septic AKI}

Contrary to the abovementioned studies, many expressions decreased lncRNAs could also participate in critical pathophysiological processes in septic AKI. PlncRNA-1 was downregulated in the serum of patients with septic AKI and LPS-induced cells. PlncRNA-1 overexpression relived kidney injury by increasing proliferation and inhibiting apoptosis and autophagy in LPS-treated cells (Fu et al., 2018). LncRNA differentiation antagonizing non-protein coding RNA (DANCR) was also decreased in the serum of AKI patients and LPS-treated HK-2 cells. This study demonstrates that septic AKI development could be alleviated by overexpressing DANCR, which comes with the miR-214 and Krüppel-like factor 6 expression declined (Zhao et al., 2020).

Overexpressing lncRNA6406 also attenuates LPS-stimulated AKI by mitigating cell inflammation, oxidative stress, and apoptosis via modulating miR-687/PTEN signaling (Liu et al., 2020). Additionally, IncRNA HOXA-AS2 exhibited protection in sepsis-engendered AKI by targeting miR-106b-5p and hindering the Wnt/ $\beta$-catenin and NF- $\mathrm{KB}$ pathways (Wu et al., 2020b). Similarly, long intergenic non-protein coding RNA261(LINC00261) could function as a sponge to combine with microRNA-654-5p, which inhibits NF- $\mathrm{kB}$ activity by 
TABLE 1 | LncRNAs in septic AKI.

\begin{tabular}{|c|c|c|c|c|c|}
\hline AKI & IncRNA & Expression & Regulation network & Function & Reference \\
\hline \multirow[t]{38}{*}{ Septic AKI } & MALAT1 & up & $\mathrm{miR}-146 a / N F-\kappa B$ & $\begin{array}{l}\text { Promote cytokine creation and immune } \\
\text { response }\end{array}$ & Ding et al. (2018) \\
\hline & NEAT1 & up & $\mathrm{miR}-22-3 p / N F-\kappa B$ & Promote cell apoptosis & Feng et al. (2020) \\
\hline & & & let-7b-5p/TRAF6 & Promote inflammation & Gao et al. (2020b) \\
\hline & & & miR-93-5p/TXNIP & $\begin{array}{l}\text { Promote apoptosis, inflammation, and } \\
\text { oxidative stress }\end{array}$ & Yang et al. (2021b) \\
\hline & & & miR-27a-3p/TAB3 & Promote cell apoptosis & Wang et al. (2020a) \\
\hline & & & miR-125a-5P/TRAF6/TAK1 & Modulate macrophage polarization & Wang and Guo (2020) \\
\hline & PVT1 & up & $\mathrm{miR}-17-5 \mathrm{p} / \mathrm{NF}-\mathrm{kB}$ & Promote cell apoptosis & Yuan et al. (2021b) \\
\hline & & & miR-20a-5p/NLRP3 & $\begin{array}{l}\text { Promote cell pyroptosis and inflammatory } \\
\text { factors creation }\end{array}$ & Deng et al. (2021b) \\
\hline & DLX6-AS1 & up & miR-223-3p/NLRP3 & Improve cytotoxicity & Tan et al. (2020) \\
\hline & MEG3 & up & miR-21/PDCD4 & Promote cell apoptosis & Yang et al. (2018) \\
\hline & & & miR-18a-3p/GSDMD & Promote pyroptosis & Deng et al. (2021a) \\
\hline & TapSAKI & up & miR-22/PTEN/TLR4/NF-кB & $\begin{array}{l}\text { Promote cell apoptosis and inflammation } \\
\text { response }\end{array}$ & Shen et al. (2019) \\
\hline & & & miR-205/IRF3 & Enhance cytotoxicity & Han et al. (2021b) \\
\hline & SNHG14 & up & miR-93/IRAK4/NF-кB & Accelerate cellular injury & Shi et al. (2021) \\
\hline & & & miR-495-3p/HIPK1 & Inhibit cell proliferation and autophagy & Yang et al. (2021a) \\
\hline & SNHG5 & up & miR-374a-3p/TLR4/NF-кB & Promote cell apoptosis & Wang et al. (2021) \\
\hline & MIAT & up & $\mathrm{miR}-29 a$ & Promote cell apoptosis & Zhang et al. (2019) \\
\hline & ANRIL & up & miR-199a/TLR4/NF-kB & Promote cell apoptosis & Zhu et al. (2020b) \\
\hline & TCONS_00016233 & up & miR-22-3p/AIFM1 & $\begin{array}{l}\text { Promote LPS-induced HK-2 cell apoptosis } \\
\text { and the expression of IL-1b and TNF-a }\end{array}$ & Zhang et al. (2020b) \\
\hline & SIKIAT1 & up & miR-96-3p & Promote cell apoptosis & Lu et al. (2020b) \\
\hline & RMRP & up & miR-206/DPX5 & Promote cell apoptosis & Zhang et al. (2021) \\
\hline & NKILA & up & miR-140-5p/CLDMA & Promote cell apoptosis & Han et al. (2021a) \\
\hline & XIST & up & miR-15a-5p/ CUL3 & Enhance podocyte cell apoptosis & Xu et al. (2019) \\
\hline & GAS5 & down & PI3K/AKT & Promotes podocyte injury & Fang et al. (2018) \\
\hline & PIncRNA-1 & down & - & $\begin{array}{l}\text { Promote cell proliferation, inhibit apoptosis } \\
\text { and autophagy }\end{array}$ & Fu et al. (2018) \\
\hline & DANCR & down & miR-214/KLF6 & $\begin{array}{l}\text { Suppress cell apoptosis and cytokine } \\
\text { creation }\end{array}$ & Zhao et al. (2020) \\
\hline & IncRNA6406 & down & miR-687/PTEN & $\begin{array}{l}\text { Alleviate inflammation, oxidative stress, and } \\
\text { inhibit apoptosis }\end{array}$ & Liu et al. (2020) \\
\hline & HOXA-AS2 & down & miR-106b-5p/Wnt/ $\beta$-catenin/ NF-кB & Inhibit the inflammation & Wu et al. (2020b) \\
\hline & LINC00261 & down & miR-654-5p/SOCS3/NF-кB & $\begin{array}{l}\text { Improve cell viability, suppress the } \\
\text { apoptosis, and reduced the generation of } \\
\text { inflammation cytokines }\end{array}$ & Li et al. (2021c) \\
\hline & CASC2 & down & $\mathrm{miR}-155 / \mathrm{NF}-\kappa \mathrm{B}$ & $\begin{array}{l}\text { Inhibit inflammation factors creation, cell } \\
\text { apoptosis and oxidative stress }\end{array}$ & Wang et al. (2020d) \\
\hline & & & miR-545-3p/PPARA & $\begin{array}{l}\text { Facilities cell viability and restrain cell } \\
\text { apoptosis migration EMT and oxidative } \\
\text { stress }\end{array}$ & Hu et al. (2021) \\
\hline & CCAT-1 & down & miR-155/SIRT1 & $\begin{array}{l}\text { Attenuate inflammatory response and } \\
\text { apoptosis }\end{array}$ & Lu et al. (2020a) \\
\hline & NONRATG019935.2 & down & P53 & Suppress the apoptosis & Ding et al. (2021) \\
\hline & CRNDE & up & TLR3/NF-кB & Promote kidney injury & Sun et al. (2019) \\
\hline & & & $\mathrm{miR}-146 \mathrm{a} / \mathrm{TLR} 4 / \mathrm{NF}-\kappa \mathrm{B}$ & $\begin{array}{l}\text { Accelerate LPS-induced inflammation and } \\
\text { apoptosis }\end{array}$ & Wu et al. (2020a) \\
\hline & & down & miR-181a-5p & $\begin{array}{l}\text { Accelerate LPS-induced inflammation and } \\
\text { apoptosis }\end{array}$ & Wang et al. (2020c) \\
\hline & HOTAIR & up & miR-34a/Bcl-2 & Inhibit the apoptosis of kidney tissues & Jiang et al. (2019b) \\
\hline & & & miR-22/HMGB1 & Promote HK-2 cell apoptosis & Shen et al. (2018) \\
\hline
\end{tabular}

targeting the suppressor of cytokine signaling 3 (Li et al., 2021c). Long noncoding RNA cancer susceptibility candidate 2 (CASC2) has also been shown to protect against sepsis induced AKI by blocking the miR155 and NF- $\kappa B$ signaling pathways (Wang et al., 2020d). Furthermore, CASC2 overexpression facilitated cell viability and restrained cell apoptosis, migration, epithelial-mesenchymal transition (EMT), and oxidative stress through regulating the miR-545-3p/PPARA axis in LPS-triggered HK-2 and HEK293 cells (Hu et al., 2021).

LncRNA Colon cancer-associated transcript-1 (CCAT1) was shown to have anti-inflammation and pro-survival effects in LPS induced renal tubular epithelial cell damage in vitro and in vivo. This study also revealed the mechanism of dysregulated CCAT1/miR-155/SIRT1 pathway regulated disease progression 
TABLE 2 | LncRNAs in I/R-induced AKI.

\begin{tabular}{|c|c|c|c|c|c|}
\hline AKI & IncRNA & Expression & Regulation network & Function & Reference \\
\hline \multirow[t]{15}{*}{ I/R-AKI } & MALAT1 & up & $N F-\kappa B$ & Inhibit the hypoxia-induced inflammatory response & Kölling et al. (2018) \\
\hline & PRINS & up & RANTES & Promote inflammation & Yu et al. (2016) \\
\hline & SNHG14 & up & miR-124-3p/ MMP2 & Promote inflammation and oxidative stress & Xue et al. (2021) \\
\hline & EGOT & down & HuR-ATG7/16L1 & Promote the hypoxia-induced autophagy & Wang et al. (2020b) \\
\hline & XIST & up & miR-124-3p/ITGB1 & Promote apoptosis and inflammation & Chen et al. (2020) \\
\hline & & & miR-142-5p/ PDCD4 & Promote $\mathrm{CoCl} 2$-induced cellular apoptosis & Tang et al. (2020) \\
\hline & NEAT1 & up & miR-27a-3p & Promote $\mathrm{CoCl} 2$-induced cellular apoptosis & Jiang et al. (2019a) \\
\hline & GAS5 & up & $\mathrm{miR}-21$ & Promote cell apoptosis & Geng et al. (2020) \\
\hline & LINC00520 & up & miR-27b-3p/OSMR & Promote cell apoptosis & Tian et al. (2019) \\
\hline & LINC00963 & up & miR-128-3p/ JAK2/STAT1 & Reduce G1 arrest and apoptosis & Xie et al. (2020) \\
\hline & MEG3 & up & miR-145-5p /Wnt/ $\beta$-catenin & Activate mitophagy and induce apoptosis & Liu et al. (2021) \\
\hline & LINC00052 & down & miR-532-3p/ Wnt/ $\beta$-catenin & Decrease hypoxia-induced ROS and MDA accumulation & Li et al. (2020) \\
\hline & $\mathrm{H} 19$ & up & miR-130a/BCL2L11 & Augment cell apoptosis & Yuan et al. (2021a) \\
\hline & & & miR-30a-5p & Reduce cell apoptosis and inflammation & Haddad et al. (2021) \\
\hline & TUG1 & down & miR-494-3p/ E-cadherin & Alleviate cell apoptosis & Chen et al. (2021b) \\
\hline
\end{tabular}

in a murine model of LPS-induced AKI (Lu et al., 2020a). A recent study identified LncRNA NONRATG019935.2 exhibited a significant reduction both in septic AKI rats and LPS-treated NRK-52E cells. Similarly, overexpression of NONRATG019935.2 suppressed cell apoptosis and p53 protein levels in LPS-treated NRK-52E cells and retarded septic AKI development in the rat model of septic AKI (Ding et al., 2021).

\section{LncRNAs Showed a Bidirectional Effect on Septic AKI}

Several lncRNAs also showed a bidirectional effect on septic AKI in the current study. For example, LPS treatment increased ncRNA colorectal neoplasia differentially expressed gene (CRNDE) expression in HK-2 cells and mice. CRNDE inhibition could reduce sepsis-induced kidney injury by blocking the activation of the TLR3/NF-kB pathway (Sun et al., 2019). Meanwhile, overexpressing CRNDE activated TLR4/NF- $\kappa B$ signaling by regulating miR-146a, which accelerated LPS-induced inflammation and apoptosis in HK-2 cells (Wu et al., 2020a). In contrast, the opposite results have been found in the urine-derived septic rats, and in LPS-treated HK-2 and HEK293 cells, CRNDE was markedly down-regulated. Down-regulation of CRNDE could aggravate kidney injury via increasing miR-181a-5p (Wang et al., 2020c). Another lncRNA with a dual effect in septic AKI is lncRNA HOX transcript antisense RNA (HOTAIR). Overexpression of HOTAIR inhibited apoptosis by downregulating miR-34a/ Bcl-2 signaling in the cecal ligation puncture septic AKI rats (Jiang et al., 2019b). However, in the urine-derived sepsis rat model, HOTAIR was up-regulated, and it promoted HK-2 cell apoptosis through the miR-22/HMGB1 pathway (Shen et al., 2018).

\section{LNCRNAS IN I/R INDUCED AKI}

\section{LncRNAs Changed Globally in I/R Induced AKI}

Ischemia-reperfusion is one of the leading causes of AKI. Inflammation and oxidative stress are the most common processes involved in the pathogenesis of renal I/R injury. Zhou and coworkers profiled the lncRNA expression pattern in the kidney of AKI mice by second-generation gene sequencing. They reported 90 differentially expressed lncRNAs which included 20 up-regulated and 70 down-regulated. These dysregulated lncRNAs participated in multiple biological processes, including stimulus response, multicellular organismal processes, singlemulticellular organism processes, and so on (Zhou et al., 2017a). In 2017, a study reported 52 differentially expressed lncRNAs in the kidney, where three significantly up-regulated lncRNAs (TCONS_00042175, TCONS_00058568, and TCONS_00047728) were negatively correlated with I/R-induced kidney injury (Liu et al., 2017). Another study revealed the altered ncRNA in I/R-induced AKI, which covered the change of lncRNAs and showed the lncRNA-miRNA-mRNA regulatory network based on ceRNA theory. In a recent study, Tao et al. also reported that a total of 2,267 lncRNAs were prominently expressed in experimental I/R mice (Tao et al., 2019).

\section{LncRNAs Promoting I/R Induced AKI}

Studies have been conducted to determine if lncRNAs are implicated in I/R-induced AKI (Table 2). Hypoxia contributes to renal functional decline during I/R injury. LncRNA MALAT1 was activated by hypoxia-inducible factor $1-\alpha$ (HIF-1 $\alpha$ ) and negatively regulated the expression of IL- 6 , TNF- $\alpha$, and NF-kB (Kölling et al., 2018). Another HIF-1 $\alpha$ dependent lncRNAs psoriasis susceptibility-related RNA gene induced by stress (Prins) has been shown to increase in I/R damaged kidney and interact with RANTES to mediate various inflammatory responses (Yu et al., 2016).

In vitro experiments, the lncRNA SNHG14 could mitigate I/R-induced kidney injury via competition with miR-124-3p to regulate the expression of MMP2 in HK-2 cells (Xue et al., 2021). The decline of IncRNA eosinophil granule ontogeny transcript (EGOT) promoted hypoxia-induced autophagy in HK-2 cells via binding with the RNA-binding protein $\mathrm{Hu}$ antigen $\mathrm{R}(\mathrm{HuR})$ and regulation of ATG7/16L1 expression (Wang et al., 2020b). The other three expressions increased lncRNA X-inactive 
specific transcript (XIST), NEAT1, and GAS5 also promoted cell apoptosis and inflammation through the modulation of miR-124-3p, miR-27a-3p, and miR-21, separately (Jiang et al., 2019a; Chen et al., 2020; Geng et al., 2020). Meanwhile, other researchers have experimentally validated the upregulation of lncRNA XIST in I/R-AKI and showed its regulatory role of miR-142-5p and PDCD4 (Tang et al., 2020). Signaling pathway analysis was performed by mapping AKI related lncRNAs to KEGG pathways. One study reported that lncRNA LINC00520/ miR-27b-3p/OSMR axis contributed to the AKI aggravation by activating the PI3K/AKT pathway (Tian et al., 2019). LINC00963, another IncRNA interplay with miR-128-3p, could promote AKI through the JAK2/STAT1 pathway (Xie et al., 2020). The lncRNA MEG3 has also been found to trigger the Wnt/ $\beta$-catenin pathway to aggravate kidney I/R injury (Liu et al., 2021).

\section{LncRNAs Exerted Nephroprotective Effects on I/R-AKI}

So far, only one lncRNA has been found to have a protective effect on I/R-AKI. The lncRNA LINC00052 expression was significantly decreased in AKI patient plasma but enormously elevated in hypoxic cells. Overexpression of LINC00052 could ameliorate AKI by reactivating Wnt/ $\beta$-catenin signaling, which is inactivated in NRK-52E cells under hypoxic conditions via sponging miR-532-3p (Li et al., 2020).

\section{LncRNAs Showed a Bidirectional Effect on I/R-AKI}

LncRNA H19 and lncRNA TUG1 harbored a bidirectional regulation of cellular function in IR-induced AKI. The lncRNA H19 could regulate the miR-130a/BCL2L11 axis to modulate the proliferation and apoptosis of HK-2 cells under hypoxia/ reoxygenation conditions. In parallel, downregulation of lncRNA H19 could promote cell proliferation, inhibit cell apoptosis, and suppress inflammatory cytokine expression in HK-2 cells throughout the miR-130a/BCL2L11 pathway (Yuan et al., 2021a). However, another study has found a contrary function of H19 in I/R-AKI. George et al. pointed out that overexpression of lncRNA H19 in the I/R model could provide a significant renal-protective effect through sponging of microRNA-30a-5p (Haddad et al., 2021). Overexpressing lncRNA TUG1 diminished the protective effect of total glucosides of paeony on AKI and exacerbated autophagy in HK-2 cells (Chang et al., 2021b). However, Chen et al. found that overexpression of lncRNA TUG1 could significantly alleviate cell apoptosis by serving as a miR-494-3p sponge to disinhibit E-cadherin (Chen et al., 2021b).

\section{LNCRNAS IN POST-CONTRAST AKI}

Post-contrast acute kidney injury (PC-AKI), also called contrastinduced acute kidney injury (CI-AKI), is a kind of kidney disease that the renal function deteriorates suddenly within $48 \mathrm{~h}$ of intravascular administration of contrast medium (van der Molen et al., 2018). Data have shown that PC-AKI was the third most common cause of AKI in hospitalized patients (Nash et al., 2002) and accounted for high mortality rates (Levy et al., 1996). More recently, the literature has emerged that offers significant findings of the definition, risk factors, pathophysiology mechanism, and medical intervention of PC-AKI (Mehran et al., 2019; Bansal and Patel, 2020; Everson et al., 2020). Bioinformatics analysis revealed that lncRNAs could serve as novel biomarkers in the early phase of PC-AKI (Table 3). Chen et al. depicted the expression landscape of lncRNAs and identified 357 differentially expressed lncRNAs in PC-AKI. Among these, lnc-HILPDA and lnc-PRND, which were conservative and remarkably up-regulated in both kidneys and blood from rats and the blood of PC-AKI patients, can effectively predict PC-AKI risk and precisely distinguish PC-AKI patients after the exposure to contrast medium (Chen et al., 2021a).

Since the expression of lncRNAs has undergone massive changes in PC-AKI, more and more researchers are delving into the specific regulatory mechanism that lncRNA is involved in PC-AKI. Cheng and coworkers systematically explored the lncRNAassociated-ceRNA regulatory network in rats with PC-AKI by RNA-seq analysis and two constructed ceRNA regulatory pathways in the CI-AKI rat model (novel_circ_0004153/rnomiR-144-3p/ Gpnmb or Naglu and LNC_000343/rno-miR-1956-5p/KCP) were reported firstly and validated by real-time qPCR. Further function annotation shows that the disease-specific $\ln \mathrm{CRNAs}$ may participate in physiological and pathological processes of PC-AKI from different aspects, including porphyrin-containing compound metabolic, biosynthetic processes, heme binding, and so on (Cheng et al., 2019). Another study also found and verified that differential expressed IncRNAs and their co-expression targets were associated with vital pathological pathways of PC-AKI, like inflammation and oxidative stress (Bao et al., 2021). For instance, they identified a group of lncRNAs (such as MSTRG.11448.4, MSTRG.2420.1, MSTRG.6245.1, and NONRATT023367.2) linked to glycoprotein non-metastatic melanoma protein, which can negatively regulate inflammation in AKI. Furthermore, IncRNA-associated ceRNA analysis revealed a Cndp1-specific network that was also likely to be related to the process of antioxidation, which plays a vital role in oxidative stress-mediated injury in PC-AKI (Ripoll et al., 2007; Zhou et al., 2017b). In summary, although hundreds of lncRNAs associated explicitly with the PC-AKI have been identified and several studies have constructed their regulatory networks with other RNAs, the specific biological functions and detailed mechanisms of the vast majority of lncRNAs remain unclear, and further research in this area is required.

\section{LNCRNA IN KIDNEY TRANSPLANT ACUTE KIDNEY INJURY}

Kidney transplantation is one of the effective treatments for patients with end-stage renal disease (ESRD). However, as a potentially severe complication, post-transplant AKI still maintains high morbidity and mortality rates (Mittal and Kohli, 2014). The pathogenesis of post-transplant AKI is complex. Several researchers have found the association between lncRNA and post-transplant AKI (Table 3). 
TABLE 3 | LncRNAs in PC-AKI, post-transplant AKI, and cisplatin induced AKI.

\begin{tabular}{|c|c|c|c|c|c|}
\hline AKI & LncRNA & Expression & Regulation network & Function & Reference \\
\hline \multirow[t]{3}{*}{ PC-AKI } & LNC_000343 & down & rno-miR-1956-5p/KCP & Reduce interstitial fibrosis and renal injury & Cheng et al. (2019) \\
\hline & NONRATT025462.2 & down & $\begin{array}{l}\text { rno-miR-126a-5p, miR-200a- } \\
\text { 5p, miR-200a-5p/Cndp1 }\end{array}$ & Associated with the process of antioxidation & Bao et al. (2021) \\
\hline & NONRATT020679.2 & down & $\begin{array}{l}\text { rno-miR-126a-5p, miR-200a- } \\
\text { 5p/Tmem184b }\end{array}$ & - & Bao et al. (2021) \\
\hline \multirow[t]{3}{*}{ Post-transplant AKI } & ATB & up & $\mathrm{miR}-200 \mathrm{c} / \mathrm{TGF}-\beta$ & $\begin{array}{l}\text { Activate cell proliferation and cyclosporine } \\
\text { A-mediated apoptosis }\end{array}$ & Qiu et al. (2017) \\
\hline & XIST & - & miR-212-3p/ ASF1A, BRWD1 & Regulate inflammatory and apoptosis & Cheng and Wang (2020) \\
\hline & & & miR-122-5p/ PFKFB2 & $\begin{array}{l}\text { Influence thyroid hormone and AMPK } \\
\text { signaling }\end{array}$ & Cheng and Wang (2020) \\
\hline \multirow[t]{6}{*}{ Cisplatin induced AKI } & LncRNA 9,884 & up & $\begin{array}{l}\mathrm{NF}-\kappa \mathrm{B} / \text { macrophage migration } \\
\text { inhibitory factor }\end{array}$ & Aggravate tubular epithelial cells injury & Zhang et al. (2020a) \\
\hline & MEG3 & up & AKT/TSC/mTOR & Promote cisplatin-induced nephrotoxicity & Jing et al. (2021) \\
\hline & GAS5 & up & miR-205-5p & Aggravate renal epithelial cell apoptosis & Zhang et al. (2021) \\
\hline & OIP5-AS1 & down & miR-144-5p/PKM2 & Reduce the apoptosis of renal epithelial cells & Chang et al. (2021a) \\
\hline & XLOC_032768 & down & $\mathrm{TNF}-\alpha$ & Reduce cell apoptosis & Zhou et al. (2020) \\
\hline & PRNCR1 & down & miR-182-5p/EZH1 & Reduce cell apoptosis & Li et al. (2021a) \\
\hline
\end{tabular}

In 2013, Sui et al. integrated protein array-based proteomics and RNA microarray-based genomics to investigate the transcription factors (TF), miRNAs, long noncoding RNA of biopsies of three patients with acute rejections. They discovered that among the 32 differentially expressed lncRNAs, FXYD1 and HOXA11 were targeted individually by the lncRNAs Uc002nyb and Uc003syy, which were linked to a poor outcome in acute rejections (Sui et al., 2013). Another study also used lncRNA microarrays to compare the lncRNA expression in kidney biopsy from acute rejection following kidney transplantation. After validating the microarray results by qRT-PCR and screening in the five databases, the functions of five candidate IncRNAs (AF113674, uc003wbj, uc010ftb, uc001fty, and AK129917) were further defined by their location to the gene on the genome (Chen et al., 2014). Other studies have investigated the correlation between post-transplant AKI-specific lncRNA expression and the diagnosis or prognosis of acute rejection. LncRNA AF264622 and AB209021 exhibited excellent diagnostic performance both in pediatric and adult renal transplants (Ge et al., 2017). Furthermore, IncRNA MIR155HG, which participates in allograft rejection associated pathways, such as graft versus host disease, T-cell and B cell receptor, signaling pathways, can predict the risk of graft loss effectively (Zou et al., 2019).

Notably, lncRNAs are also released and circulated in the plasma, urine, and other body fluids. Investigators have also found lncRNAs in blood or urine that could be used for early detection of post-transplant AKI (Lorenzen et al., 2015; Ge et al., 2017). However, studies describing the mechanism of lncRNA in post-transplant AKI are limited. LncRNA-ATB, a novel lncRNA activated by TGF- $\beta$, may contribute to kidney transplantation survival through mediating the TGF- $\beta$ signaling pathway. In this study, the expression of IncRNA-ATB was largely up-regulated in the TGF- $\beta$ treated human tubule cells, activating cell proliferation and cyclosporine A-mediated apoptosis of renal cells via the downregulation of miR-200c (Qiu et al., 2017). Another up-regulated lncRNA in the serum of a post-transplant patient with AKI is XIST which could serve as a ceRNA to sponge hsa-miR-212-3p to regulate inflammation and apoptosis in the progression of AKI via modulating the expression of ASF1A and BRWD1. Furthermore, lncRNA XIST could also sponge miR-122-5p to influence thyroid hormone and AMPK signaling via modulating the expression of PFKFB2 in AKI (Cheng and Wang, 2020).

Overall, these studies reasonably provide consistent evidence of an association between lncRNA and post-transplant AKI. Most current research on lncRNA remains at the level of their expression pattern identification and diagnostic or prognostic potential in diseases. There is still much room for improvement, and more attention should be paid to their therapeutic potential.

\section{LNCRNA OF AKI INDUCED BY OTHER CAUSES}

Aside from the four types of AKI mentioned above, medication or toxin-induced AKI is a kind of AKI that is common in the clinic but poorly understood in lncRNA-based biological mechanism research. Upon closer inspection of the literature, We identified only six relevant papers investigating the role of lncRNA in cisplatin reduced AKI. At the same time, no study reports the relationship between IncRNAs in other common drug-induced AKI, such as aristolochic acid, folic acid, and antibiotics for the time being (Table 3). Among them, lncRNA9884 is a promoting factor of cisplatin induced AKI. Expression elevated lncRNA9884 could significantly aggravate tubular epithelial cells injury by activating inflammatory signaling pathway NF- $\kappa \mathrm{B} /$ macrophage migration inhibitory factor (Zhang et al., 2020a). Another recent study has described that the lncRNA MEG3 could promote cisplatin-induced nephrotoxicity through regulating AKT/TSC/mTOR-mediated autophagy, and LncRNA GAS5 could aggravate renal epithelial cell apoptosis in cisplatin-induced AKI by regulating miR-205-5p (Jing et al., 2021; Zhang et al., 2021). 


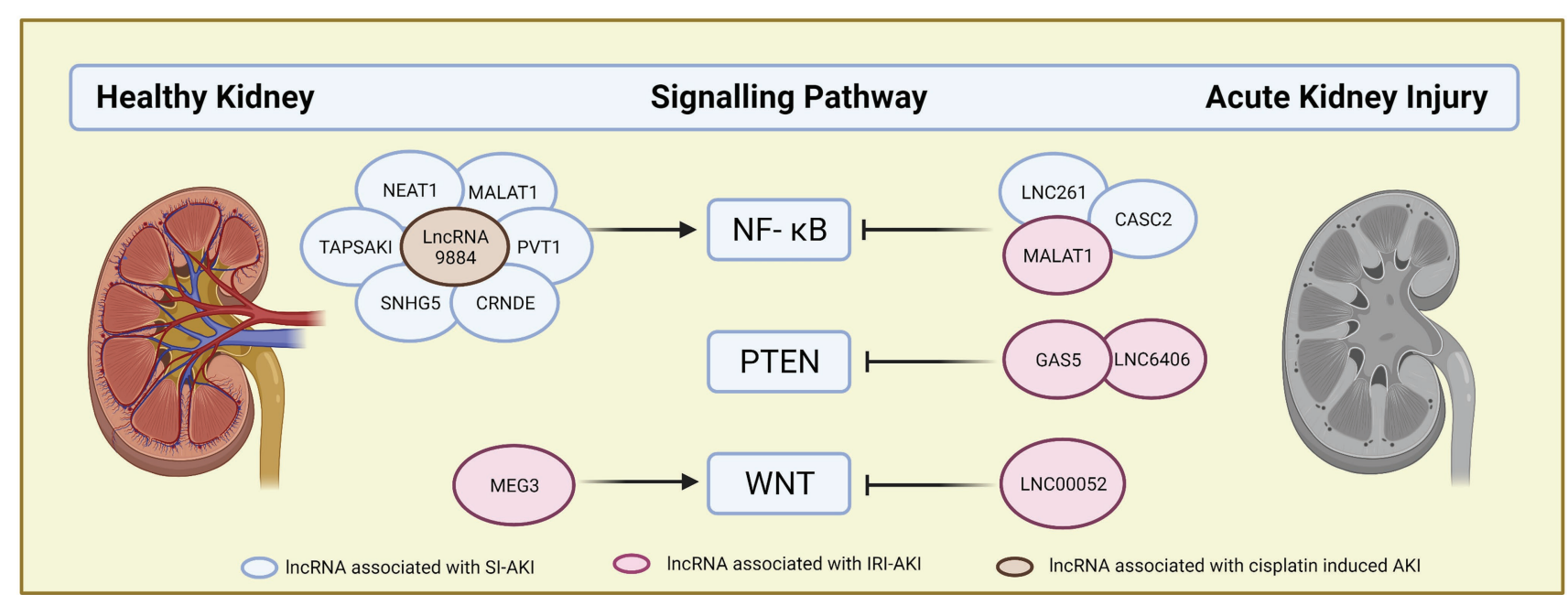

FIGURE 2 | Canonical IncRNAs signaling pathway. LncRNAs are summarized by their function in key signaling pathways in AKI. LncRNAs on the left are the IncRNA which could activate the signaling pathway, while IncRNAs on the right are the IncRNA which could inactivate the signaling pathway. Different colors of circles indicate the different types of AKI. Blue circle: sepsis induced AKI, red circle: ischemic/perfusion induced AKI, brown circle: cisplatin induced AKI.

One study found that lncRNA OIP5-AS1 was significantly downregulated in cisplatin-induced AKI model both in vitro and in vivo. Further research revealed that OIP5-AS1 could reduce the apoptosis of cisplatin-stimulated renal epithelial cells to exert a protective effect by targeting the miR-144-5p/PKM2 axis (Chang et al., 2021a). Similarly, the novel lncRNA XLOC_032768 and PRNCR1 could also perform a similar role of reducing cisplatin-induced apoptosis of renal tubular epithelial cells to protect against the cisplatin-induced AKI through TNF- $\alpha$ and miR-182-5p/EZH1, respectively (Zhou et al., 2020; Li et al., 2021a). Regrettably, as for the other reasons induced AKI, for instance, rhabdomyolysis-associated AKI and trauma associated AKI, the biological mechanism of those diseases has been studied, but not for the role of IncRNAs.

\section{DISCUSSION}

LncRNAs offer a novel direction for pathophysiological study and the search for new therapeutic targets in acute kidney injury. On the one hand, lncRNAs exhibit the potential to act as biomarkers for the early diagnosis and prognosis of patients with AKI. On the other hand, lncRNAs have been identified as the key mediators that participated in the pathophysiology of AKI caused by different reasons. Expression, function, and regulation network of lncRNAs altered depending on the causes of AKI. Several identified lncRNAs, such as XIST, MALAT1, NEAT1, and so on, contribute to kidney injury in two or more different reasons caused AKI through different regulatory mechanisms. Those $\operatorname{lncRNAs}$ may represent the potential therapeutic target for the AKI. Besides, based on the ceRNA regulatory network, one lncRNA can be involved in the different signaling pathways and link with different targets downstream, which are closely related to the process of cell apoptosis, inflammation, such as NF- $\mathrm{BB}$, WNT, and PTEN (Figure 2). This feature greatly enriched and complicated the regulatory mechanism of lncRNA and provided a way to develop new therapeutic solutions for AKI.

Although the function and the diagnostic and prognostic roles of lncRNAs in AKI had been carried out extensively, the limitations are also evident in the current study. First, most studies of lncRNA function were based on the ceRNA network, which could only reveal lncRNA function at the post-translational level, possibly because of the use of bioinformatics to predict the functions of lncRNAs is preferred over traditional, time-consuming, and expensive experimental methods. Neither the function of IncRNAs at the epigenetic level or transcription level, nor the cell organelle-specific function of lncRNAs gained equitable attention in lncRNAs in AKI. Second, the specific mechanisms of various studies are still unclear, and more in-depth research is needed to reveal the relevant mechanisms. Finally, clinical trials to validate the therapeutic potential of lncRNAs in AKI are currently inadequate due to the lack of a safe and effective delivery mechanism. There is still a long way to go before the study results can be promoted clinically. For sure, some difficulties exist in the lncRNAs research due to the low expression, low sequence conservation, and diverse secondary functional structure of the lncRNAs. Thus, in the future, the more credible algorithms need to be developed, the more precise detection technology of lncRNAs needs to be innovated, the more safe and efficient drug delivery method needs to be devised. In summary, there remains a huge gap in access to a comprehensive understanding of LncRNAs in AKI, and further efforts will be needed.

\section{AUTHOR CONTRIBUTIONS}

LY and BW defined the subject, revised the literature, and wrote the manuscript. All authors contributed to the article and approved the submitted version. 


\section{FUNDING}

This study was supported by the National Key R\&D Program of China (2020YFC2005000). Figures 1 and $\mathbf{2}$ were created with a free version of Biorender.com

\section{REFERENCES}

Bansal, S., and Patel, R. N. (2020). Pathophysiology of contrast-induced acute kidney injury. Interv. Cardiol. Clin. 9, 293-298. doi: 10.1016/j.iccl.2020.03.001

Bao, W., Xiao, Z., Wang, Z., Liu, D., Tan, P., and Huang, M. (2021). Comprehensive analysis of the long non-coding RNA expression profile and functional roles in a contrast-induced acute kidney injury rat model. Exp. Ther. Med. 22:739. doi: 10.3892/etm.2021.10171

Bridges, M. C., Daulagala, A. C., and Kourtidis, A. (2021). LNCcation: IncRNA localization and function. J. Cell Biol. 220:e202009045. doi: 10.1083/ jcb. 202009045

Cao, M., Zhao, J., and Hu, G. (2019). Genome-wide methods for investigating long noncoding RNAs. Biomed. Pharmacother. 111, 395-401. doi: 10.1016/j. biopha.2018.12.078

Chang, S., Chang, M., Liu, G., Xu, D., Wang, H., Sun, R., et al. (2021a). LncRNA OIP5-AS1 reduces renal epithelial cell apoptosis in cisplatin-induced AKI by regulating the miR-144-5p/PKM2 axis. Biom. J. doi: 10.1016/j. bj.2021.07.005

Chang, X., Zhang, P., Xu, X. X., and Pang, B. (2021b). Total glucosides of paeony inhibited autophagy and improved acute kidney injury induced by ischemia-reperfusion via the lncRNA TUG1/miR-29a/PTEN Axis. Drug Des. Devel. Ther. 15, 2229-2242. doi: 10.2147/dddt.S286606

Chen, L.-L. (2016). Linking long noncoding RNA localization and function. Trends Biochem. Sci. 41, 761-772. doi: 10.1016/j.tibs.2016.07.003

Chen, F., Hu, Y., Xie, Y., Zhao, Z., Ma, L., Li, Z., et al. (2020). Total glucosides of paeony alleviate cell apoptosis and inflammation by targeting the Long noncoding RNA XIST/MicroRNA-124-3p/ITGB1 axis in renal ischemia/ reperfusion injury. Mediat. Inflamm. 2020:8869511, -8869513. doi: $10.1155 / 2020 / 8869511$

Chen, G., Liu, B., Chen, S., Li, H., Liu, J., Mai, Z., et al. (2021a). Novel biomarkers for post-contrast acute kidney injury identified from long noncoding RNA expression profiles. Int. J. Biol. Sci. 17, 882-896. doi: 10.7150/ ijbs. 45294

Chen, W., Peng, W., Huang, J., Yu, X., Tan, K., Chen, Y., et al. (2014). Microarray analysis of long non-coding RNA expression in human acute rejection biopsy samples following renal transplantation. Mol. Med. Rep. 10, 2210-2216. doi: $10.3892 / \mathrm{mmr} .2014 .2420$

Chen, L., Xu, J. Y., and Tan, H. B. (2021b). LncRNA TUG1 regulates the development of ischemia-reperfusion mediated acute kidney injury through miR-494-3p/E-cadherin axis. J Inflamm 18:12. doi: 10.1186/ s12950-021-00278-4

Cheng, W., Li, X. W., Xiao, Y. Q., and Duan, S. B. (2019). Non-coding RNAassociated ceRNA networks in a new contrast-induced acute kidney injury rat model. Mol Ther Nucleic Acids 17, 102-112. doi: 10.1016/j.omtn.2019.05.011

Cheng, Q., and Wang, L. (2020). LncRNA XIST serves as a ceRNA to regulate the expression of ASF1A, BRWD1M, and PFKFB2 in kidney transplant acute kidney injury via sponging hsa-miR-212-3p and hsa-miR-122-5p. Cell Cycle 19, 290-299. doi: 10.1080/15384101.2019.1707454

Chun-Mei, H., Qin-Min, G., Shu-Ming, P., and Xiang-Yang, Z. (2016). Expression profiling and ontology analysis of circulating long non-coding RNAs in septic acute kidney injury patients. Clin. Chem. Lab. Med. 54, e395-e399. doi: $10.1515 / \mathrm{cclm}-2015-1281$

Deng, J., Tan, W., Luo, Q., Lin, L., Zheng, L., and Yang, J. (2021a). Long non-coding RNA MEG3 promotes renal tubular epithelial cell pyroptosis by regulating the miR-18a-3p/GSDMD pathway in lipopolysaccharideinduced acute kidney injury. Front. Physiol. 12:663216. doi: 10.3389/ fphys.2021.663216

Deng, L. T., Wang, Q. L., Yu, C., and Gao, M. (2021b). IncRNA PVT1 modulates NLRP3-mediated pyroptosis in septic acute kidney injury by targeting miR-20a-5p. Mol. Med. Rep. 23:11910. doi: 10.3892/mmr.2021.11910

\section{SUPPLEMENTARY MATERIAL}

The Supplementary Material for this article can be found online at: https://www.frontiersin.org/articles/10.3389/fphys. 2022.849403/full\#supplementary-material

Derrien, T., Johnson, R., Bussotti, G., Tanzer, A., Djebali, S., Tilgner, H., et al. (2012). The GENCODE v7 catalog of human long noncoding RNAs: analysis of their gene structure, evolution, and expression. Genome Res. 22, 1775-1789. doi: $10.1101 /$ gr.132159.111

Ding, Y., Guo, F., Zhu, T., Li, J., Gu, D., Jiang, W., et al. (2018). Mechanism of long non-coding RNA MALAT1 in lipopolysaccharide-induced acute kidney injury is mediated by the miR-146a/NF- $\mathrm{BB}$ signaling pathway. Int J. Mol. Med. 41, 446-454. doi: 10.3892/ijmm.2017.3232

Ding, Y., Zhou, D.-Y., Yu, H., Zhu, T., Guo, F., He, Y., et al. (2021). Upregulation of IncRNA NONRATG019935.2 suppresses the p53-mediated apoptosis of renal tubular epithelial cells in septic acute kidney injury. Cell Death Dis. 12:771. doi: 10.1038/s41419-021-03953-9

Everson, M., Sukcharoen, K., and Milner, Q. (2020). Contrast-associated acute kidney injury. BJA Educ. 20, 417-423. doi: 10.1016/j.bjae.2020.07.006

Fang, Y., Hu, J.-F., Wang, Z.-H., Zhang, S.-G., Zhang, R.-F., Sun, L.-M., et al. (2018). GAS5 promotes podocyte injury in sepsis by inhibiting PTEN expression. Eur. Rev. Med. Pharmacol. Sci. 22, 8423-8430. doi: 10.26355/ eurrev_201812_16541

Feng, Y., Liu, J., Wu, R., Yang, P., Ye, Z., and Song, F. (2020). NEAT1 aggravates sepsis-induced acute kidney injury by sponging miR-22-3p. Open Med 15, 333-342. doi: 10.1515/med-2020-0401

Fenoglio, C., Ridolfi, E., Galimberti, D., and Scarpini, E. (2013). An emerging role for long non-coding RNA Dysregulation in neurological disorders. Int J. Mol. Sci. 14, 20427-20442. doi: 10.3390/ijms141020427

Fernandes, J. C. R., Acuña, S. M., Aoki, J. I., Floeter-Winter, L. M., and Muxel, S. M. (2019). Long non-coding RNAs in the regulation of gene expression: physiology and disease. Noncoding RNA 5:17. doi: 10.3390/ ncrna5010017

Fu, D., Zhou, K., Liu, J., Zheng, P., Li, P., Cheng, W., et al. (2018). Long non-coding RNA PlncRNA-1 regulates cell proliferation, apoptosis, and autophagy in septic acute kidney injury by regulating BCL2. Int. J. Clin. Exp. Pathol. 11, 314-323.

Gao, N, Li, Y, Li, J, Gao, Z, Yang, Z, Li, Y, et al. (2020a). Frontiers in Oncology Available at: https://www.frontiersin.org/article/10.3389/fonc.2020.598817 (Accessed January 26, 2022).

Gao, C., Zou, X., Chen, H., Shang, R., and Wang, B. (2020b). Long non-coding RNA nuclear paraspeckle assembly transcript 1 (NEAT1)relieves sepsis-induced kidney injury and lipopolysaccharide (LPS)-induced inflammation in HK-2 cells. Med. Sci. Monit. 26:e921906. doi: 10.12659/MSM.921906

Ge, Y.-Z., Xu, T., Cao, W.-J., Wu, R., Yao, W.-T., Zhou, C.-C., et al. (2017). A molecular signature of two long non-coding RNAs in peripheral blood predicts acute renal allograft rejection. Cell. Physiol. Biochem. 44, 1213-1223. doi: $10.1159 / 000485451$

Gelali, E., Girelli, G., Matsumoto, M., Wernersson, E., Custodio, J., Mota, A., et al. (2019). iFISH is a publically available resource enabling versatile DNA FISH to study genome architecture. Nat. Commun. 10:1636. doi: 10.1038/ s41467-019-09616-w

Geng, X., Song, N., Zhao, S., Xu, J., Liu, Y., Fang, Y., et al. (2020). LncRNA GAS5 promotes apoptosis as a competing endogenous RNA for miR-21 via thrombospondin 1 in ischemic AKI. Cell Death Dis. 6:19. doi: 10.1038/ s41420-020-0253-8

Haddad, G., Kölling, M., Wegmann, U. A., Dettling, A., Seeger, H., Schmitt, R., et al. (2021). Renal AAV2-mediated overexpression of long non-coding RNA H19 attenuates ischemic acute kidney injury through sponging of microRNA-30a-5p. J. Am. Soc. Nephrol. 32, 323-341. doi: 10.1681/ASN.2020060775

Han, D., Fang, R., Shi, R., Jin, Y., and Wang, Q. (2021a). LncRNA NKILA knockdown promotes cell viability and represses cell apoptosis, autophagy and inflammation in lipopolysaccharide-induced sepsis model by regulating miR-140-5p/CLDN2 axis. Biochem. Biophys. Res. Commun. 559, 8-14. doi: 10.1016/j.bbrc.2021.04.074 
Han, X., Yuan, Z., Jing, Y., Zhou, W., Sun, Y., and Xing, J. (2021b). Knockdown of IncRNA TapSAKI alleviates LPS-induced injury in HK-2 cells through the miR-205/IRF3 pathway. Open Med 16, 581-590. doi: 10.1515/med-2021-0204

Hernández, H. G., Tse, M. Y., Pang, S. C., Arboleda, H., and Forero, D. A. (2013). Optimizing methodologies for PCR-based DNA methylation analysis. BioTechniques 55, 181-197. doi: 10.2144/000114087

Hu, Q., Zen, W., Zhang, M., Wang, Z., Cui, W., Liu, Y., et al. (2021). Long non-coding RNA CASC2 overexpression ameliorates sepsis-associated acute kidney injury by regulating MiR-545-3p/PPARA axis. J. Surg. Res. 265, 223-232. doi: 10.1016/j.jss.2021.03.047

Huang, W., Li, X., Wang, D., Sun, Y., Wang, Q., Bu, Y., et al. (2020). Curcumin reduces LPS-induced septic acute kidney injury through suppression of lncRNA PVT1 in mice. Life Sci. 254:117340. doi: 10.1016/j.lfs.2020.117340

Jiang, X., Li, D., Shen, W., Shen, X., and Liu, Y. (2019a). LncRNA NEAT1 promotes hypoxia-induced renal tubular epithelial apoptosis through downregulating miR-27a-3p. J. Cell. Biochem. 120, 16273-16282. doi: 10.1002/ jcb.28909

Jiang, Z. J., Zhang, M. Y., Fan, Z. W., Sun, W. L., and Tang, Y. (2019b). Influence of IncRNA HOTAIR on acute kidney injury in sepsis rats through regulating miR-34a/Bcl-2 pathway. Eur. Rev. Med. Pharmacol. Sci. 23, 3512-3519. doi: 10.26355/eurrev_201904_17717

Jing, X., Han, J., Zhang, J., Chen, Y., Yuan, J., Wang, J., et al. (2021). Long non-coding RNA MEG3 promotes cisplatin-induced nephrotoxicity through regulating AKT/TSC/mTOR-mediated autophagy. Int. J. Biol. Sci. 17, 3968-3980. doi: $10.7150 /$ ijbs.58910

Kellum, J. A., Lameire, N., Aspelin, P., Barsoum, R. S., Burdmann, E. A., Goldstein, S. L., et al. (2012). Kidney disease: improving global outcomes (KDIGO) acute kidney injury work group. KDIGO clinical practice guideline for acute kidney injury. Kidney Int. Suppl. 2:1. doi: 10.1038/kisup.2012.1

Kinsey, G. R., and Okusa, M. D. (2011). Pathogenesis of acute kidney injury: foundation for clinical practice. Am. J. Kidney Dis. 58, 291-301. doi: 10.1053/j. ajkd.2011.02.385

Kölling, M., Genschel, C., Kaucsar, T., Hübner, A., Rong, S., Schmitt, R., et al. (2018). Hypoxia-induced long non-coding RNA Malat1 is dispensable for renal ischemia/reperfusion-injury. Sci. Rep. 8:3438. doi: 10.1038/ s41598-018-21720-3

Lan, C., Peng, H., Hutvagner, G., and Li, J. (2019). Construction of competing endogenous RNA networks from paired RNA-seq data sets by pointwise mutual information. BMC Genomics 20:943. doi: 10.1186/s12864-019-6321-x

Levy, E. M., Viscoli, C. M., and Horwitz, R. I. (1996). The effect of acute renal failure on mortality. A cohort analysis. JAMA 275, 1489-1494. doi: 10.1001/jama.1996.03530430033035

Lewington, A. J. P., Cerdá, J., and Mehta, R. L. (2013). Raising awareness of acute kidney injury: a global perspective of a silent killer. Kidney Int. 84, 457-467. doi: 10.1038/ki.2013.153

Li, J., Fan, X., Wang, Q., Gong, Y., and Guo, L. (2021a). Long noncoding RNA PRNCR1 reduces renal epithelial cell apoptosis in cisplatin-induced AKI by regulating miR-182-5p/EZH1. Kidney Blood Press. Res. 46, 162-172. doi: $10.1159 / 000510157$

Li, Y., Li, W., Hoffman, A. R., Cui, J., and Hu, J.-F. (2021b). The nucleus/ mitochondria-shuttling LncRNAs function as new epigenetic regulators of mitophagy in cancer. Front. Cell Dev. Biol. 9:699621. doi: 10.3389/ fcell.2021.699621

Li, X., Li, J., Lu, P., and Li, M. (2021c). LINC00261 relieves the progression of sepsis-induced acute kidney injury by inhibiting NF- $\mathrm{kB}$ activation through targeting the miR-654-5p/SOCS3 axis. J. Bioenerg. Biomembr. 53, 129-137. doi: $10.1007 / \mathrm{s} 10863-021-09874-8$

Li, X., Zheng, P., Ji, T., Tang, B., Wang, Y., and Bai, S. (2020). LINC00052 ameliorates acute kidney injury by sponging miR-532-3p and activating the Wnt signaling pathway. Aging (Albany NY) 13, 340-350. doi: 10.18632/ aging.104152

Liu, Q. Q., Liu, H., He, Z. G., Zhang, S. J., Liu, B. W., Wang, L., et al. (2017). Differential gene and lncRNA expression in the lower thoracic spinal cord following ischemia/reperfusion-induced acute kidney injury in rats. Oncotarget 8, 53465-53481. doi: 10.18632/oncotarget.18584

Liu, D., Liu, Y., Zheng, X., and Liu, N. (2021). C-MYC-induced long noncoding RNA MEG3 aggravates kidney ischemia-reperfusion injury through activating mitophagy by upregulation of RTKN to trigger the Wnt/ $\beta$-catenin pathway. Cell Death Dis. 12:191. doi: 10.1038/s41419-021-03466-5
Liu, F., Zheng, K., Chen, H.-C., and Liu, Z.-F. (2018). Capping-RACE: a simple, accurate, and sensitive 5' RACE method for use in prokaryotes. Nucleic Acids Res. 46:e129. doi: 10.1093/nar/gky739

Liu, X., Zhu, N., Zhang, B., and Xu, S. B. (2020). Long noncoding RNA TCONS_00016406 attenuates lipopolysaccharide-induced acute kidney injury by regulating the miR-687/PTEN pathway. Front. Physiol. 11:622. doi: 10.3389/ fphys.2020.00622

Lorenzen, J. M., Schauerte, C., Kölling, M., Hübner, A., Knapp, M., Haller, H., et al. (2015). Long noncoding RNAs in urine are detectable and may enable early detection of acute $\mathrm{T}$ cell-mediated rejection of renal allografts. Clin. Chem. 61, 1505-1514. doi: 10.1373/clinchem.2015.243600

Lu, S., Dong, L., Jing, X., Gen-Yang, C., and Zhan-Zheng, Z. (2020a). Abnormal lncRNA CCAT1/microRNA-155/SIRT1 axis promoted inflammatory response and apoptosis of tubular epithelial cells in LPS caused acute kidney injury. Mitochondrion 53, 76-90. doi: 10.1016/j.mito.2020.03.010

Lu, S., Wu, H., Xu, J., He, Z., Li, H., and Ning, C. (2020b). SIKIAT1/miR-96/ FOXA1 axis regulates sepsis-induced kidney injury through induction of apoptosis. Inflamm. Res. 69, 645-656. doi: 10.1007/s00011-020-01350-0

Ma, T., Jia, H., Ji, P., He, Y., and Chen, L. (2021). Identification of the candidate lncRNA biomarkers for acute kidney injury: a systematic review and metaanalysis. Expert. Rev. Mol. Diagn. 21, 77-89. doi: 10.1080/14737159.2021.1873131

Marchese, F. P., Raimondi, I., and Huarte, M. (2017). The multidimensional mechanisms of long noncoding RNA function. Genome Biol. 18:206. doi: 10.1186/s13059-017-1348-2

Mehran, R., Dangas, G. D., and Weisbord, S. D. (2019). Contrast-associated acute kidney injury. N. Engl. J. Med. 380, 2146-2155. doi: 10.1056/ NEJMra1805256

Mehta, R. L., Cerdá, J., Burdmann, E. A., Tonelli, M., García-García, G., Jha, V., et al. (2015). International Society of Nephrology's 0by25 initiative for acute kidney injury (zero preventable deaths by 2025): a human rights case for nephrology. Lancet 385, 2616-2643. doi: 10.1016/S0140-6736(15)60126-X

Mercer, T. R., Dinger, M. E., and Mattick, J. S. (2009). Long non-coding RNAs: insights into functions. Nat. Rev. Genet. 10, 155-159. doi: 10.1038/nrg2521

Mittal, T., and Kohli, H. S. (2014). Post renal transplant acute kidney injury. Ind. J. Transpl. 8, S33-S36. doi: 10.1016/j.ijt.2014.01.011

Nakato, R., and Sakata, T. (2021). Methods for ChIP-seq analysis: a practical workflow and advanced applications. Methods 187, 44-53. doi: 10.1016/j. ymeth.2020.03.005

Nash, K., Hafeez, A., and Hou, S. (2002). Hospital-acquired renal insufficiency. Am. J. Kidney Dis. 39, 930-936. doi: 10.1053/ajkd.2002.32766

Peng, W.-X., Koirala, P., and Mo, Y.-Y. (2017). LncRNA-mediated regulation of cell signaling in cancer. Oncogene 36, 5661-5667. doi: 10.1038/onc.2017.184

Qiu, J., Chen, Y., Huang, G., Zhang, Z., Chen, L., and Na, N. (2017). Transforming growth factor- $\beta$ activated long non-coding RNA ATB plays an important role in acute rejection of renal allografts and may impacts the postoperative pharmaceutical immunosuppression therapy. Nephrology (Carlton) 22, 796-803. doi: $10.1111 /$ nep. 12851

Ripoll, V. M., Irvine, K. M., Ravasi, T., Sweet, M. J., and Hume, D. A. (2007). Gpnmb is induced in macrophages by IFN-gamma and lipopolysaccharide and acts as a feedback regulator of proinflammatory responses. J. Immunol. 178, 6557-6566. doi: 10.4049/jimmunol.178.10.6557

Salviano-Silva, A., Lobo-Alves, S. C., de Almeida, R. C., Malheiros, D., and Petzl-Erler, M. L. (2018). Besides pathology: long non-coding RNA in cell and tissue homeostasis. Noncoding RNA 4:3. doi: 10.3390/ncrna4010003

Shen, J., Liu, L., Zhang, F., Gu, J., and Pan, G. (2019). LncRNA TapSAKI promotes inflammation injury in HK-2 cells and urine derived sepsisinduced kidney injury. J. Pharm. Pharmacol. 71, 839-848. doi: 10.1111/ jphp.13049

Shen, J., Zhang, J., Jiang, X., Wang, H., and Pan, G. (2018). LncRNA HOX transcript antisense RNA accelerated kidney injury induced by urine-derived sepsis through the miR-22/high mobility group box 1 pathway. Life Sci. 210, 185-191. doi: 10.1016/j.lfs.2018.08.041

Shi, C., Zhao, Y., Li, Q., and Li, J. (2021). IncRNA SNHG14 plays a role in sepsis-induced acute kidney injury by regulating miR-93. Mediat. Inflamm. 2021:5318369. doi: $10.1155 / 2021 / 5318369$

Statello, L., Guo, C. J., Chen, L. L., and Huarte, M. (2021). Gene regulation by long non-coding RNAs and its biological functions. Nat. Rev. Mol. Cell Biol. 22, 96-118. doi: 10.1038/s41580-020-00315-9 
Sui, W., Lin, H., Peng, W., Huang, Y., Chen, J., Zhang, Y., et al. (2013). Molecular dysfunctions in acute rejection after renal transplantation revealed by integrated analysis of transcription factor, microRNA and long noncoding RNA. Genomics 102, 310-322. doi: 10.1016/j.ygeno.2013.05.002

Sun, B. Q., Sui, Y. D., Huang, H., Zou, X. B., Chen, S. C., and Yu, Z. K. (2019). Effect of IncRNA CRNDE on sepsis-related kidney injury through the TLR3/NF-кB pathway. Eur. Rev. Med. Pharmacol. Sci. 23, 10489-10497. doi: 10.26355/eurrev_201912_19688

Tan, J., Fan, J., He, J., Zhao, L., and Tang, H. (2020). Knockdown of LncRNA DLX6-AS1 inhibits HK-2 cell pyroptosis via regulating miR-223-3p/NLRP3 pathway in lipopolysaccharide-induced acute kidney injury. J. Bioenerg. Biomembr. 52, 367-376. doi: 10.1007/s10863-020-09845-5

Tang, B., Li, W., Ji, T., Li, X., Qu, X., Feng, L., et al. (2020). Downregulation of XIST ameliorates acute kidney injury by sponging miR-142-5p and targeting PDCD4. J. Cell. Physiol. 235, 8852-8863. doi: 10.1002/ jcp.29729

Tao, Q., Tianyu, W., Jiangqiao, Z., Zhongbao, C., Xiaoxiong, M., Long, Z., et al. (2019). Expression analysis of long non-coding RNAs in a renal ischemia-reperfusion injury model. Acta Cir. Bras. 34:e201900403. doi: 10.1590/ s0102-865020190040000003

Tian, X., Ji, Y., Liang, Y., Zhang, J., Guan, L., and Wang, C. (2019). LINC00520 targeting miR-27b-3p regulates OSMR expression level to promote acute kidney injury development through the PI3K/AKT signaling pathway. J. Cell. Physiol. 234, 14221-14233. doi: 10.1002/jcp.28118

van der Molen, A. J., Reimer, P., Dekkers, I. A., Bongartz, G., Bellin, M.-F., Bertolotto, M., et al. (2018). Post-contrast acute kidney injury - part 1: definition, clinical features, incidence, role of contrast medium and risk factors. Eur. Radiol. 28, 2845-2855. doi: 10.1007/s00330-017-5246-5

Wang, J., Chen, Y., Tang, Z., Hu, D., Yao, C., and Yang, L. (2020a). LncRNA NEAT1 regulated inflammation and apoptosis in a rat model of sepsisinduced acute kidney injury via MiR-27a-3p/TAB3 axis. Biosci. Biotechnol. Biochem. 84, 2215-2227. doi: 10.1080/09168451.2020.1792760

Wang, W., and Guo, Z. H. (2020). Downregulation of lncRNA NEAT1 ameliorates LPS-induced inflammatory responses by promoting macrophage M2 polarization via miR-125a-5p/TRAF6/TAK1 Axis. Inflammation 43, 1548-1560. doi: $10.1007 /$ s10753-020-01231-y

Wang, I.-K., Palanisamy, K., Sun, K.-T., Yu, S.-H., Yu, T.-M., Li, C.-H., et al. (2020b). The functional interplay of lncRNA EGOT and HuR regulates hypoxia-induced autophagy in renal tubular cells. J. Cell. Biochem. 121, 4522-4534. doi: 10.1002/jcb.29669

Wang, J., Song, J., Li, Y., Shao, J., Xie, Z., and Sun, K. (2020c). Downregulation of LncRNA CRNDE aggravates kidney injury via increasing MiR-181a-5p in sepsis. Int. Immunopharmacol. 79:105933. doi: 10.1016/j. intimp.2019.105933

Wang, M., Wei, J., Shang, F., Zang, K., and Ji, T. (2020d). Long non-coding RNA CASC2 ameliorates sepsis-induced acute kidney injury by regulating the miR-155 and NF-кB pathway. Int. J. Mol. Med. 45, 1554-1562. doi: 10.3892/ijmm.2020.4518

Wang, M., Wei, J., Shang, F., Zang, K., and Zhang, P. (2021). Down-regulation of lncRNA SNHG5 relieves sepsis-induced acute kidney injury by regulating the miR-374a-3p/TLR4/NF-kB pathway. J. Biochem. 169, 575-583. doi: 10.1093/ $\mathrm{jb} / \mathrm{mvab} 008$

Wu, S., Qiu, H., Wang, Q., Cao, Z., and Wang, J. (2020a). Effects and mechanism of lncRNA CRNDE on sepsis-induced acute kidney injury. Anal. Cell. Pathol. 2020:8576234, -8576238. doi: 10.1155/2020/8576234

Wu, H., Wang, J., and Ma, Z. (2020b). Long noncoding RNA HOXA-AS2 mediates microRNA-106b-5p to repress sepsis-engendered acute kidney injury. J. Biochem. Mol. Toxicol. 34:e22453. doi: 10.1002/jbt.22453

Xie, L. B., Chen, B., Liao, X., Chen, Y. F., Yang, R., He, S. R., et al. (2020). LINC00963 targeting miR-128-3p promotes acute kidney injury process by activating JAK2/STAT1 pathway. J. Cell. Mol. Med. 24, 5555-5564. doi: $10.1111 / \mathrm{jcmm} .15211$

Xu, L., Hu, G., Xing, P., Zhou, M., and Wang, D. (2020). Paclitaxel alleviates the sepsis-induced acute kidney injury via lnc-MALAT1/miR-370-3p/HMGB1 axis. Life Sci. 262:118505. doi: 10.1016/j.lfs.2020.118505

Xu, G., Mo, L., Wu, C., Shen, X., Dong, H., Yu, L., et al. (2019). The miR-15a-5p-XIST-CUL3 regulatory axis is important for sepsis-induced acute kidney injury. Ren. Fail. 41, 955-966. doi: 10.1080/0886022X.2019.1669460
Xue, Q., Yang, L., Wang, H., and Han, S. (2021). Silence of Long noncoding RNA SNHG14 alleviates ischemia/reperfusion-induced acute kidney injury by regulating miR-124-3p/MMP2 Axis. Biomed. Res. Int. 2021:8884438, -8884413. doi: 10.1155/2021/8884438

Yang, R., Liu, S., Wen, J., Xue, L., Zhang, Y., Yan, D., et al. (2018). Inhibition of maternally expressed gene 3 attenuated lipopolysaccharide-induced apoptosis through sponging miR-21 in renal tubular epithelial cells. J. Cell. Biochem. 119, 7800-7806. doi: 10.1002/jcb.27163

Yang, N., Wang, H., Zhang, L., Lv, J., Niu, Z., Liu, J., et al. (2021a). Long non-coding RNA SNHG14 aggravates LPS-induced acute kidney injury through regulating miR-495-3p/HIPK1. Acta Biochim. Biophys. Sin. Shanghai 53, 719-728. doi: 10.1093/abbs/gmab034

Yang, J., Wu, L., Liu, S., Hu, X., Wang, Q., and Fang, L. (2021b). Long non-coding RNA NEAT1 promotes lipopolysaccharide-induced injury in human tubule epithelial cells by regulating miR-93-5p/TXNIP axis. Med. Microbiol. Immunol. 210, 121-132. doi: 10.1007/ s00430-021-00705-6

Yu, T. M., Palanisamy, K., Sun, K. T., Day, Y. J., Shu, K. H., Wang, I. K., et al. (2016). RANTES mediates kidney ischemia reperfusion injury through a possible role of HIF- $1 \alpha$ and LncRNA PRINS. Sci. Rep. 6:18424. doi: $10.1038 /$ srep 18424

Yuan, Y., Li, X., Chu, Y., Ye, G., Yang, L., and Dong, Z. (2021a). Long noncoding RNA H19 augments hypoxia/Reoxygenation-induced renal tubular epithelial cell apoptosis and injury by the miR-130a/BCL2L11 pathway. Front. Physiol. 12:632398. doi: 10.3389/fphys.2021.632398

Yuan, W., Xiong, X., Du, J., Fan, Q., Wang, R., and Zhang, X. (2021b). LncRNA PVT1 accelerates LPS-induced septic acute kidney injury through targeting

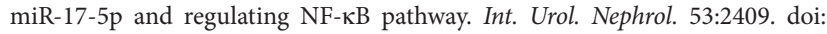
10.1007/s11255-021-02905-8

Zhang, X., Huang, Z., Wang, Y., Wang, T., Li, J., and Xi, P. (2021). Long non-coding RNA RMRP contributes to sepsis-induced acute kidney injury. Yonsei Med. J. 62, 262-273. doi: 10.3349/ymj.2021.62.3.262

Zhang, Y., Tang, P. M., Niu, Y., García Córdoba, C. A., Huang, X. R., Yu, C., et al. (2020a). Long non-coding RNA LRNA9884 promotes acute kidney injury via regulating NF-kB-mediated transcriptional activation of MIF. Front. Physiol. 11:590027. doi: 10.3389/fphys.2020.590027

Zhang, P., Yi, L., Qu, S., Dai, J., Li, X., Liu, B., et al. (2020b). The biomarker TCONS_00016233 drives septic AKI by targeting the miR-22-3p/AIFM1 signaling Axis. Mol. Ther. Nucleic Acids 19, 1027-1042. doi: 10.1016/j. omtn.2019.12.037

Zhang, Y., Zhang, Y.-Y., Xia, F., Yang, A.-X., Qian, J.-X., Zhao, H., et al. (2019). Effect of lncRNA-MIAT on kidney injury in sepsis rats via regulating miR-29a expression. Eur. Rev. Med. Pharmacol. Sci. 23, 10942-10949. doi: 10.26355/ eurrev_201912_19797

Zhao, H., Chen, B., Li, Z., Wang, B., and Li, L. (2020). Long noncoding RNA DANCR suppressed lipopolysaccharide-induced septic acute kidney injury by regulating miR-214 in HK-2 cells. Med. Sci. Monit. 26:e921822. doi: 10.12659/MSM.921822

Zhou, J., Chen, H., and Fan, Y. (2017a). Systematic analysis of the expression profile of non-coding RNAs involved in ischemia/reperfusion-induced acute kidney injury in mice using RNA sequencing. Oncotarget 8, 100196-100215. doi: 10.18632/oncotarget.22130

Zhou, X., Jiang, K., Luo, H., Wu, C., Yu, W., and Cheng, F. (2020). Novel IncRNA XLOC_032768 alleviates cisplatin-induced apoptosis and inflammatory response of renal tubular epithelial cells through TNF- $\alpha$. Int. Immunopharmacol. 83:106472. doi: 10.1016/j.intimp.2020.106472

Zhou, L., Zhuo, H., Ouyang, H., Liu, Y., Yuan, F., Sun, L., et al. (2017b). Glycoprotein non-metastatic melanoma protein b (Gpnmb) is highly expressed in macrophages of acute injured kidney and promotes M2 macrophages polarization. Cell. Immunol. 316, 53-60. doi: 10.1016/j. cellimm.2017.03.006

Zhu, S., and Lu, Y. (2020a). Dexmedetomidine suppressed the biological behavior of HK-2 cells treated with LPS by down-regulating ALKBH5. Inflammation 43, 2256-2263. doi: 10.1007/s10753-020-01293-y

Zhu, Y., Wei, S. W., Ding, A., Zhu, W. P., Mai, M. F., Cui, T. X., et al. (2020b). The long noncoding RNA ANRIL promotes cell apoptosis in lipopolysaccharide-induced acute kidney injury mediated by the TLR4/ nuclear factor-kappa B pathway. Kidney Blood Press. Res. 45, 209-221. doi: $10.1159 / 000505154$ 
Zou, Y., Zhang, W., Zhou, H. H., and Liu, R. (2019). Analysis of long noncoding RNAs for acute rejection and graft outcome in kidney transplant biopsies. Biomark. Med 13, 185-195. doi: 10.2217/bmm-2018-0272

Conflict of Interest: The authors declare that the research was conducted in the absence of any commercial or financial relationships that could be construed as a potential conflict of interest.

Publisher's Note: All claims expressed in this article are solely those of the authors and do not necessarily represent those of their affiliated organizations, or those of the publisher, the editors and the reviewers. Any product that may be evaluated in this article, or claim that may be made by its manufacturer, is not guaranteed or endorsed by the publisher.

Copyright (c) 2022 Yang, Wang, Ma and Fu. This is an open-access article distributed under the terms of the Creative Commons Attribution License (CC BY). The use, distribution or reproduction in other forums is permitted, provided the original author(s) and the copyright owner(s) are credited and that the original publication in this journal is cited, in accordance with accepted academic practice. No use, distribution or reproduction is permitted which does not comply with these terms. 